\title{
Management of Crohn's disease in poor responders to adalimumab
}

This article was published in the following Dove Press journal:

Clinical and Experimental Gastroenterology

II April 2014

Number of times this article has been viewed

\section{Nanne KH de Boer \\ Mark Löwenberg² \\ Frank Hoentjen ${ }^{3}$}

'Department of Gastroenterology and Hepatology, VU University Medical

Center, Amsterdam, the Netherlands; ${ }^{2}$ Department of Gastroenterology and Hepatology, Academic Medical Center, Amsterdam, the Netherlands; ${ }^{3}$ Department of Gastroenterology and Hepatology, Radboud University Medical Center, Nijmegen, the Netherlands
Correspondence: Frank Hoentjen Department of Gastroenterology and Hepatology, PO Box 910I, Nijmegen, the Netherlands Tel +3I 2436 I 6999

Email frankhoentjen@gmail.com

\begin{abstract}
Anti-tumor necrosis factor therapy with adalimumab is an effective therapy for the induction and maintenance of remission in moderate to severe Crohn's disease. Although a large proportion of patients show a favorable clinical response to adalimumab, therapy failure is common. In this review, we provide a practical overview of adalimumab therapy in patients with Crohn's disease, with a specific focus on the clinical management of adalimumab failure. In the case of inadequate efficacy, a thorough assessment is required to confirm inflammatory disease activity and rule out noninflammatory causes. Evaluation may include biomarkers (fecal calprotectin and serum C-reactive protein), colonoscopy, and/or magnetic resonance enterography/enteroclysis. Furthermore, adalimumab trough levels and antibodies to adalimumab are informational after the confirmation of active inflammation. In the case of low or undetectable adalimumab trough levels, dose escalation to $40 \mathrm{mg}$ weekly is recommended, whereas high antibody titers or adverse events frequently require switching to an alternative anti-TNF agent such as infliximab. Active inflammation despite therapeutic adalimumab trough levels requires alternative strategies such as switching to drugs with a different mode of action or surgical intervention.
\end{abstract}

Keywords: anti-TNF, biological, inflammatory bowel disease, loss of response, infliximab

\section{Introduction}

When treating patients with Crohn's disease, therapy is generally aimed at successful induction and subsequent maintenance of remission, as well as reducing therapyrelated complications, avoidance of (prolonged) glucocorticosteroid administration, and improved quality of life. Mucosal healing and histological normalization of the inflamed intestinal mucosa is another therapeutic endpoint, as evidence suggests that mucosal healing may have a beneficial effect on the disease course. ${ }^{1}$

Anti-tumor necrosis factor (TNF) therapy is an effective therapy for Crohn's disease, and a large proportion of patients show a favorable response to these therapeutic antibodies. The first patients with Crohn's disease were successfully treated in 1995 with anti-TNF therapy. ${ }^{2}$ The development and subsequent introduction in clinical practice of the anti-TNF agents infliximab (which received US Food and Drug Administration [FDA] approval in 1998) and adalimumab (which received FDA approval in 2007) has led to an important broadening of the therapeutic arsenal for treating Crohn's disease patients. Despite therapeutic efficacy of anti-TNF agents, treatment failure is commonly observed, and in recent years, significant progress has been made in optimizing the clinical management of loss of response to these agents. In this review, we provide a practical overview of adalimumab therapy in Crohn's 
disease patients, with a specific focus on the clinical management of adalimumab failure.

\section{Pharmacology of adalimumab}

Adalimumab is a fully humanized, recombinant, monoclonal immunoglobulin G1 antibody that targets TNF. Adalimumab binds with high affinity and specificity to TNF, leading to inhibition of the interaction between TNF and its cell surface TNF receptor, thereby neutralizing the inflammatory effects of TNF. After a single subcutaneous administration of $40 \mathrm{mg}$ adalimumab in healthy volunteers, the maximal serum concentration was observed after approximately 5 days. The average absolute bioavailability of adalimumab was $64 \%$, and the mean terminal half-life is approximately 14 days. $^{3}$ The mean serum concentration of adalimumab after the induction phase $(160 \mathrm{mg}$ at week 0 followed by $80 \mathrm{mg}$ at week 2 ) was approximately $13 \mu \mathrm{g} / \mathrm{mL} .{ }^{4}$ In patients with rheumatoid arthritis, adalimumab levels are influenced by concomitant methotrexate administration, as those patients receiving combination therapy display higher median adalimumab concentrations. ${ }^{5}$ However, concomitant therapy with thiopurines did not lead to an important alteration in adalimumab serum concentrations in Crohn's disease patients. ${ }^{4}$ Conversely, adalimumab therapy also has no influence on thiopurine metabolism, as demonstrated by a prospective pharmacokinetic study in 12 patients with Crohn's disease. ${ }^{6}$

As adalimumab is a humanized therapeutic protein, antibodies against adalimumab may be generated over time. In general, the presence of these antidrug antibodies has been associated with an increased risk for adverse events and reduced therapeutic efficacy as, for example, demonstrated for infliximab. ${ }^{7}$ There is evidence to suggest that administration of adalimumab leads to a lower rate of anti-drug-antibody formation compared with the chimeric monoclonal antibody infliximab. In the Clinical Assessment of Adalimumab Safety and Efficacy Studied as an Induction Therapy in Crohn's II (CLASSIC II) trial, investigating the efficacy and safety of maintenance adalimumab therapy in comparison with placebo, only $2.6 \%(n=7)$ of patients developed antibodies against adalimumab. Interestingly, two of these seven patients (29\%) who had detectable anti-adalimumab antibodies in this trial were in clinical remission at week $56 .{ }^{8}$ In a singlecenter study from Leuven, Belgium, that investigated the efficacy of adalimumab therapy after previous infliximab failure, discontinuation of adalimumab was related to low adalimumab trough levels; ${ }^{9}$ in addition, low adalimumab trough levels were more often detected in patients with detectable antibodies against adalimumab. ${ }^{9}$ The potential clinical significance of the pharmacokinetics of adalimumab trough levels and antidrug antibodies has been underlined by a cross-sectional study in 40 Crohn's disease patients that investigated mucosal healing outcomes during adalimumab therapy..$^{10}$ Adalimumab trough levels were significantly higher in patients who were in clinical remission and in those showing mucosal healing at endoscopy. Moreover, the presence of antibodies against adalimumab was predictive of not achieving mucosal healing at endoscopy. ${ }^{10}$

\section{Clinical evidence of adalimumab efficacy}

Several large, randomized controlled trials have been crucial for establishing the efficacy of adalimumab therapy in Crohn's disease for both induction as well as maintenance of remission. These trials provide insight into adalimumab failure and its management and will be briefly summarized for this purpose.

The CLASSIC I trial was designed as a Phase III, dose-ranging induction trial for adalimumab in moderate to severe Crohn's disease. ${ }^{4}$ In this randomized, double-blind, placebo-controlled trial, 299 anti-TNF-naïve subjects were randomized to receive adalimumab (weeks 0 and 2) at $160 / 80 \mathrm{mg}, 80 / 40 \mathrm{mg}, 40 / 20 \mathrm{mg}$, or placebo. After 4 weeks, remission rates (Crohn's Disease Activity Index [CDAI] score, $<150$ points, primary endpoint) were $36 \%, 24 \%, 18 \%$, and $12 \%$ for $160 / 80 \mathrm{mg}, 80 / 40 \mathrm{mg}, 40 / 20 \mathrm{mg}$, and placebo, respectively. The highest-dosing groups combined (160/80 and $80 / 40 \mathrm{mg})$ showed statistical significance $(P=0.004)$ compared with placebo. Differences in remission rates were greater in adalimumab-treated patients versus placebo when C-reactive protein (CRP) level is $1 \mathrm{mg} / \mathrm{dL}$ or higher compared with the subgroup with CRP levels lower than $1.0 \mathrm{mg} / \mathrm{dL}$. Mean serum concentrations of adalimumab were $12.61 \pm 5.25,5.65 \pm 3.06$, and $2.79 \pm 1.48 \mu \mathrm{g} / \mathrm{mL}$ for the $160 / 80$ $\mathrm{mg}, 80 / 40 \mathrm{mg}$, and $40 / 20 \mathrm{mg}$ groups, respectively.

Subsequently, in a small, Phase II maintenance study (the CLASSIC II trial), all patients from the CLASSIC I trial who achieved and maintained remission with open-label adalimumab $40 \mathrm{mg}$ every other week (eow) for an additional 4 weeks $(\mathrm{n}=55)$ were re-randomized to receive adalimumab $40 \mathrm{mg}$ eow, $40 \mathrm{mg}$ weekly, or placebo for 56 weeks. ${ }^{11}$ Patients not in remission at either time entered the open-label phase and received adalimumab $40 \mathrm{mg}$ eow $(\mathrm{n}=204)$. The primary endpoint was maintenance of clinical remission (defined as CDAI $<150$ points) in randomized patients through week 56 . At week 56 , clinical remission rates were $79 \%, 83 \%$, and 
$44 \%$ for the $40 \mathrm{mg}$ eow, $40 \mathrm{mg}$ weekly, and placebo groups, respectively. Of the remaining 204 patients who entered the open-label cohort, 131 patients completed the 56-week treatment protocol. Remission and response rates (clinical response was defined as a decrease in CDAI of at least 100 points) at week 56 in the open-label cohort were $46 \%$ and $65 \%$. The small patient number $(n=18-19)$ in the randomized group, as well as the lack of a placebo group in the openlabel cohort, limited the ability to draw firm conclusions from this trial.

Therefore, a larger Phase III, randomized, double-blind, placebo-controlled study was conducted to investigate the efficacy of adalimumab maintenance therapy in patients with moderate to severe Crohn's disease (the Crohn's Trial of the Fully Human Antibody Adalimumab for Remission Maintenance [CHARM] $).{ }^{12}$ Patients received adalimumab induction therapy $(80 / 40 \mathrm{mg})$ at weeks 0 and 2 and were subsequently randomized to receive $40 \mathrm{mg}$ eow, $40 \mathrm{mg}$ weekly, or placebo through week 56 . The primary endpoint was the rate of randomized responders (defined as CDAI decrease $\geq 70$ points at week 4 ) who achieved clinical remission (CDAI $<150$ points) at weeks 26 and 56. At baseline, $49.6 \%$ of enrolled subjects had a history of anti-TNF exposure. Among the randomized responders (499; 58\%), remission rates were $40 \%, 47 \%$, and $17 \%$ at week 26 and $36 \%, 41 \%$, and $12 \%$ at week 56 for adalimumab $40 \mathrm{mg}$ eow, $40 \mathrm{mg}$ weekly, and placebo, respectively $(P<0.001$ for each dose versus placebo). Remission rates were higher in anti-TNF-naïve patients compared with anti-TNF exposed subjects: 42\% (40 mg eow) and 48\% (40 mg weekly) of patients in the anti-TNF-naïve group were in clinical remission at week 56 versus 31\% (40 mg eow) and 34\% (40 mg weekly) in the anti-TNF-experienced group. In an open-label extension study of CHARM, called the Additional LongTerm Dosing with Humira to Evaluate Sustained Remission and Efficacy in Crohn's Disease trial (ADHERE trial), the effect of 2-year adalimumab maintenance therapy on fistula healing was studied. ${ }^{13}$ Significant and complete healing of draining fistulas was observed in adalimumab-treated patients (59.5\% [22/37]), and long-term healing of draining fistulas was maintained over the course of 2 years. The concomitant usage of antibiotics had no additional effect on the healing of fistulas in this study. However, a Dutch randomized and placebo-controlled trial comparing the efficacy of combination therapy of adalimumab and ciprofloxacin (12 weeks) with adalimumab alone in treating perianal fistulas showed that the combination was more effective (clinical response, $71 \%$ versus $47 \%) .{ }^{14}$ Of note, after cessation of ciprofloxacin, the beneficial effect of the initial coadministration was not maintained over time.

In the Gauging Adalimumab Efficacy in Infliximab Nonresponders (GAIN) trial, efficacy of adalimumab induction therapy in patients with prior infliximab failure was investigated. ${ }^{8}$ In this 4 -week randomized, double-blind, placebo-controlled trial, patients were eligible in case of moderate to severe Crohn's disease and disease activity despite infliximab treatment or intolerance to infliximab. Patients were randomly assigned to either adalimumab 160/80 mg at weeks 0 and 2 or placebo. The primary endpoint was induction of clinical remission (CDAI $<150$ points). A total of 325 subjects were randomized, and at week 4, clinical remission and response (CDAI decrease $>70$ points) rates were $21 \%$ and $52 \%$ (adalimumab) versus $7 \%$ and $34 \%$ (placebo), respectively. When remission rates in the adalimumab group were stratified according to loss of response $(20 \%)$ or intolerance to infliximab (22\%), or to the presence $(22 \%)$ or absence $(22 \%)$ of anti-infliximab antibodies, no significant differences were observed. We can deduce from this landmark study that patients who previously responded to infliximab but discontinued this therapy may benefit from a switch to adalimumab irrespective of the underlying reason for cessation of infliximab.

\section{Dosing of adalimumab therapy}

The greatest remission rates with adalimumab have been achieved with initiation of a loading dose of 160/80 mg. As outlined earlier, the CLASSIC I trial showed remission rates at week 4 of $36 \%$ with the $160 / 80 \mathrm{mg}$ induction regimen and $24 \%$ for the $80 / 40 \mathrm{mg}$ induction in anti-TNF-naïve patients. ${ }^{4}$ In patients who were previously treated with infliximab, remission rates with the $160 / 80 \mathrm{mg}$ induction dosing were greater compared with those for placebo $(21 \%$ versus $7 \%$; $P<0.001)^{8}$

The standard maintenance dosage of adalimumab is $40 \mathrm{mg}$ eow, which is largely based on the outcomes of the CHARM study, demonstrating no significant differences between $40 \mathrm{mg}$ weekly and eow at week $56 .^{12}$

\section{Primary nonresponse to adalimumab Definition and incidence}

Patients who do not respond to adalimumab induction therapy with a reduction in clinical signs and symptoms are considered primary nonresponders. ${ }^{15}$ In general, rates of primary nonresponse are variable because of differences in trial methods and cohorts but also because of a lack of 
uniform definitions. Factors such as timing of assessment after induction therapy as well as definition of clinical response vary greatly among clinical trials and explain, at least in part, the range of reported primary nonresponse rates. ${ }^{16}$ In larger controlled clinical trials such as CLASSIC I, CHARM, and GAIN, approximately $40 \%$ of patients were considered primary nonresponders. ${ }^{4,8,12}$ In uncontrolled series evaluating anti-TNF therapy, primary nonresponse rates tend to be lower. ${ }^{17}$ For example, primary nonresponse, defined as discontinuation of adalimumab and already assessed at week 4 of the induction scheme, was approximately $30 \%$ in an observational cohort of Crohn's disease patients who failed infliximab. ${ }^{9}$

\section{Causes and risk factors for primary nonresponse}

Several mechanisms underlying primary nonresponse to anti-TNF therapy have been proposed. The inflammatory mechanism can be mediated by other inflammatory cascades than TNF. This, in turn, can be dependent on, for example, the genetic background of the patient or a later disease phase. Support for the latter hypothesis comes from the observation that a shorter duration of disease is associated with higher response rates to anti-TNF therapy. ${ }^{16,18}$ In the CHARM trial, remission rates were approximately $60 \%$ in patients who had Crohn's disease for less than 2 years compared with $40 \%$ $(P<0.05)$ in patients who had a longer duration of disease. ${ }^{12}$ Pharmacokinetic factors such as drug elimination, drug binding, and antidrug antibodies may play a role as well in the mechanism of primary nonresponse.

Risk factors for primary nonresponse include the small bowel extent of disease, smoking, longer disease duration, and normal CRP levels. ${ }^{16,18}$ Although genetic risk factors have been proposed, the evidence is currently insufficient to be of relevance in clinical practice. ${ }^{18}$ Most studies that addressed risk factors for primary nonresponse assessed the response to infliximab, and studies in adalimumab nonresponders are required to improve our understanding in this matter.

\section{Management}

Prolonged administration of adalimumab beyond the proposed induction phase of 4 weeks may be applied in patients who do not have a clinical effect after the loading dose of 160/80 mg. Indeed, initial nonresponders in the CHARM study showed an increase in clinical remission rates when adalimumab was continued $(26 \%$ by week 8 and $28 \%$ by week 12 ). ${ }^{12}$ There is no solid evidence that continuation of adalimumab eow for a period longer than
12 weeks is of benefit in patients who do not demonstrate any clinical response.

If clinical response remains absent despite adequate adalimumab induction therapy, persistent disease activity should be confirmed. Clinical symptoms at baseline are frequently the result of noninflammatory causes in which case anti-TNF therapy is unlikely to provide clinical benefit. Recommendations for the diagnostic evaluation are discussed in the next sections. After confirmation of disease activity, a switch in therapeutic strategy will usually be required. Traditionally, primary nonresponse to anti-TNF therapy is considered a class effect with poor benefit from switching within the same drug class. Thus, switching to a different class of drugs, such as other immunosuppressives or integrin inhibitors such as natalizumab and vedolizumab, ${ }^{19,20}$ is considered appropriate. However, recent reports showed, in small cohorts, clinical benefit in some patients who switched to adalimumab after primary nonresponse to infliximab,,$^{21,22}$ but no evidence is yet available that supports the switch to infliximab for primary nonresponders to adalimumab. In a small series of 7 Crohn's disease patients with only partial response to adalimumab and subsequent discontinuation, none showed benefit from switching to infliximab. ${ }^{23}$

\section{Secondary nonresponse to adalimumab Definition and incidence of secondary nonresponse}

After successful induction of remission after adalimumab therapy, recurrence of symptoms can occur (so-called secondary nonresponse). This phenomenon also suffers from a lack of clear definition, but in general, secondary nonresponse implies recurrence of inflammatory disease activity. Most controlled trials define clinical remission as CDAI lower than 150 points and clinical response as a reduction in CDAI of 70 points or more from baseline. Thus, not achieving these goals 6-12 weeks after the start of induction therapy with initial response is a more specific definition of secondary nonresponse. ${ }^{17}$ Noninflammatory symptoms mimicking relapse of disease activity can negatively affect secondary nonresponse rates. Suspected secondary nonresponse is, especially in less recent studies, not always endoscopically confirmed. In daily practice, adalimumab dose escalation is a practical surrogate marker for secondary nonresponse. Despite variations in definitions, approximately $40 \%$ of initial responders to anti-TNF therapy lose response over time. ${ }^{12,24,25}$ For adalimumab, reported 
secondary nonresponse rates in controlled clinical trials range from $21 \%$ to $46 \%{ }^{8,12}$

\section{Evaluation of secondary nonresponse}

Traditionally, clinical symptoms such as abdominal pain or diarrhea were used to guide the therapeutic decisionmaking process. Initial trials that evaluated efficacy of adalimumab therapy, such as CLASSIC I, CLASSIC II, and CHARM, used a CDAI score lower than 150 points to define remission. ${ }^{4,11,12}$ However, multiple studies have underlined the poor correlation between clinical symptoms and inflammatory burden. For example, endoscopic disease activity showed a relatively good correlation with serum (CRP) and fecal (calprotectin, lactoferrin) biomarkers, but not with CDAI in Crohn's disease patients. ${ }^{26}$ Furthermore, other conditions such as irritable bowel syndrome and bile acid malabsorption can mimic Crohn's disease symptoms in the absence of inflammation. Thus, more objective measures of disease activity are required if loss of response to anti-TNF agents is suspected.

Serum CRP is an accurate marker of inflammation and correlates well with disease activity and relapses in Crohn's disease. ${ }^{27}$ Furthermore, an elevated CRP serum level 12 weeks after the start of adalimumab treatment was associated with a poor response to therapy and predicted absence of mucosal healing in Crohn's disease patients. ${ }^{28}$ A second biomarker that is frequently used to assess disease activity despite adalimumab therapy is fecal calprotectin. This noninvasive biomarker of mucosal inflammation may accurately predict a relapse of Crohn's disease..$^{29}$ An elevated calprotectin level after adalimumab induction therapy also predicted the absence of clinical remission at 12 months. It was shown that only $38 \%$ of inflammatory bowel disease patients with an elevated calprotectin level after adalimumab induction therapy achieved clinical remission at 12 months, in contrast to $84 \%$ achieving clinical remission after normalization of calprotectin levels. ${ }^{30}$

Infectious causes of diarrhea should be ruled out before further analysis. If stool analyses reveal pathogens, antibiotic therapy should be initiated accordingly. Infectious episodes and antibiotics can trigger relapses of Crohn's disease, and in the case of persistent symptoms, further diagnostic work-up should be considered.

Despite the accuracy of biomarkers, such as CRP and fecal calprotectin, to predict Crohn's disease activity, a colonoscopy remains the gold standard for diagnostic evaluation. Endoscopic evaluation can assess mucosal inflammation and allows for mucosal biopsies. A colonoscopy can also identify complications of Crohn's disease, such as strictures that can cause symptoms in the absence of inflammation. Additional imaging should be considered in the case of incomplete endoscopic assessment, the suspicion of proximal small bowel disease, or discrepancies between biomarkers and colonoscopy. Magnetic resonance enterography or enteroclysis is the preferred imaging method that can identify active inflammation as well as complications of the disease, such as fistulas, abscesses, or stenosis.

If diagnostic evaluation does not identify inflammation, a relapse of Crohn's disease is unlikely, and alternative causes should be considered. Common causes that can mimic Crohn's disease activity include irritable bowel syndrome and bile salt malabsorption, but other explanations can be considered as well on an individual basis, such as lactose intolerance, small bowel bacterial overgrowth, and celiac disease. Alternatively, noninflammatory complications of Crohn's disease can be diagnosed by endoscopy or imaging. For example, a stricture can cause obstructive symptoms, and in this case, escalation of medical therapy is unlikely to result in clinical improvement, especially when a fibrotic stricture is present without active inflammation.

If ongoing intestinal inflammation is confirmed and infections are ruled out, therapeutic management requires optimization. Trough levels and antibodies to adalimumab can guide this process (Figure 1). The timing for measuring trough levels and antidrug antibodies is variable. In the case of a high likelihood of disease activity, measurement can take place during the ongoing diagnostic evaluation to accelerate therapeutic optimization. If the likelihood of inflammatory disease activity is low, measurement can also be scheduled, depending on diagnostic outcomes. There is currently no evidence available to support routine measurements when patients maintain a state of clinical remission.

Several studies explored the use of trough levels and antibodies to predict clinical outcomes with infliximab therapy. For example, in the Study of Immunomodulator Naïve Patients in Crohn's Disease (SONIC trial), Crohn's disease patients with infliximab and trough levels higher than $1 \mu \mathrm{g} / \mathrm{mL}$ had a higher chance of achieving steroid-free clinical remission compared with those with lower infliximab trough levels. ${ }^{31}$ The detection of antibodies to infliximab correlated with a shorter duration of response and a higher rate of infusion reactions for Crohn's disease patients receiving infliximab. ${ }^{7}$ Data from adalimumab trials are more conflicting. The CLASSIC I and CLASSIC II trials were analyzed to explore a possible correlation between adalimumab trough levels and clinical remission. ${ }^{32}$ Mean adalimumab trough levels were higher 


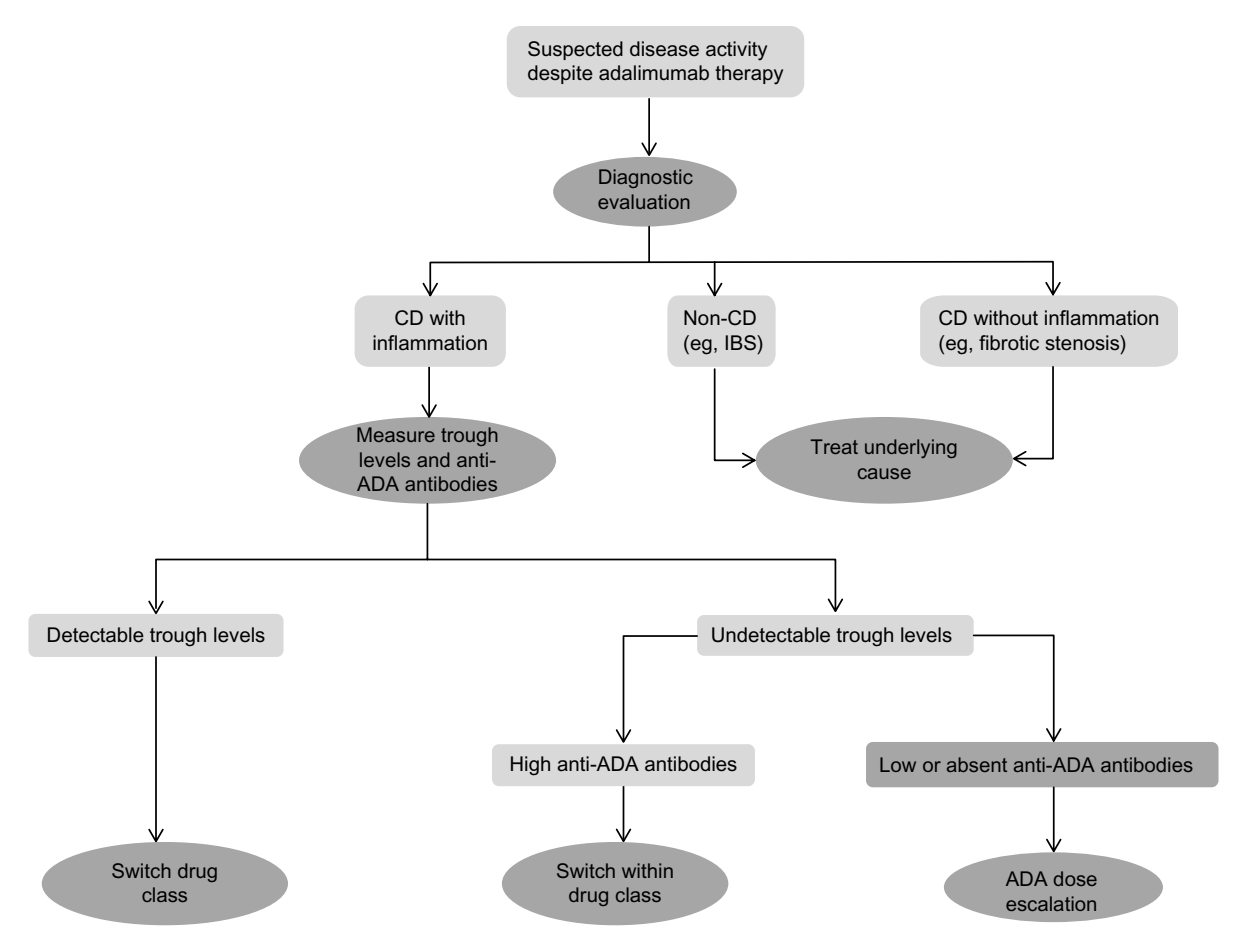

Figure I Suggested algorithm for the management of loss of response to adalimumab in Crohn's disease patients. Abbreviations: $C D$, Crohn's disease; ADA, adalimumab; IBS, irritable bowel syndrome.

in patients who achieved clinical remission at week 4 in the CLASSIC I trial compared with patients without clinical remission $(8.10$ versus $5.05 \mu \mathrm{g} / \mathrm{mL} ; P<0.05$ ). However, the variability in adalimumab concentrations was high in this study, and no cutoff concentration that predicted remission could be identified. A single-center cohort from Leuven of Crohn's disease patients receiving adalimumab treatment after infliximab failure showed lower mean trough levels $(3.2 \mu \mathrm{g} / \mathrm{mL})$ for patients who discontinued therapy compared with for patients who were able to continue therapy beyond 24 weeks. ${ }^{9}$

How trough levels and antibodies to infliximab can guide optimization of infliximab therapy was outlined in a study by Afif et al..$^{33}$ This study showed that measurement of trough levels and antibodies to infliximab affected therapeutic management in $73 \%$ of tests. In the case of low or undetectable infliximab trough levels, dose escalation was successful in $86 \%$ of patients. In patients who developed antibodies to infliximab, $92 \%$ responded favorably to a switch in therapy, whereas only $17 \%$ showed benefit from infliximab dose escalation. ${ }^{33}$ Comparable studies that focus on optimization of adalimumab therapy are lacking, although it is likely that similar algorithms can be used in clinical practice.

\section{Adjustment of therapy after secondary nonresponse}

The preferred strategy in secondary nonresponders to adalimumab is the optimization of therapy before switching to alternative anti-inflammatory agents. First, the approved options for alternative biological therapy for Crohn's disease are limited, and most patients might already have failed infliximab before starting adalimumab. Second, switching to an alternative anti-TNF agent is generally associated with a reduced response rate when compared with anti-TNF-naïve patients.

In the case of secondary nonresponse accompanied by low or undetectable trough levels, adherence to adalimumab therapy should be evaluated. Nonadherence can have various causes, including adverse events and beliefs about medication. Overall adherence to adalimumab for Crohn's disease is calculated at $83 \% .{ }^{34}$ Improving adherence is a cost-effective intervention and should be used when appropriate.

Adalimumab dose escalation is a frequently used strategy to regain clinical response. This strategy can be employed empirically in the case of increasing symptoms at the end of an injection cycle or if laboratory support for measuring trough levels and antibodies to adalimumab is not available. However, measurement of trough levels and antidrug antibodies, if available, is recommended because limited benefit from dose escalation can be expected if trough levels are in a therapeutic range or if high titers of antibodies to adalimumab are present.

Following an empirical strategy, dose escalation was required for $26 \%$ of subjects in the CHARM trial by the 
end of follow-up. ${ }^{12,35}$ A systematic review calculated that $37 \%$ of primary responders require dose intensification for loss of response, whereas the annual risk for adalimumab dose escalation is approximately $25 \%{ }^{36}$ The latter review also calculated the rate of regaining clinical response after dose escalation; this percentage was $71.4 \%$. In a single-center cohort from Leuven, $60 \%$ of initial responders to adalimumab required dose escalation during a median follow-up of 20 months, which provided clinical benefit in $75.7 \%$ of participants. ${ }^{9}$ This study also nicely illustrated that dose escalation from $40 \mathrm{mg}$ eow to $40 \mathrm{mg}$ weekly increased mean trough levels from 4.8 to $9.4 \mu \mathrm{g} / \mathrm{mL}$. This increase in trough level correlated well with clinical response. Responders displayed an increase in median trough levels of $5.9 \mu \mathrm{g} / \mathrm{mL}$ compared with $0.0 \mu \mathrm{g} / \mathrm{mL}$ for nonresponders to dose escalation. ${ }^{9}$ Collectively, these data show that adalimumab dose escalation is a successful strategy to regain clinical response that at least in part is mediated through optimization of trough levels. Of note, a nationwide retrospective Belgian study evaluated the effect of subsequent dose de-escalation to $40 \mathrm{mg}$ eow. This strategy was attempted in $54 \%$ and was successful in $63 \%$ of participants. ${ }^{37}$

Surprisingly, rates of clinical remission were similar between subjects with a detectable trough level and those with an undetectable trough level at weeks 24 and 56 of the CLASSIC II trial. ${ }^{32}$ Indeed, a similar observation was made in the SONIC trial: The steroid-free remission rate among subjects without detectable infliximab trough levels was relatively high $(59 \%){ }^{31}$ It is possible that peak adalimumab concentrations in patients who maintain clinical remission despite undetectable trough levels is sufficient to maintain their clinical state. Otherwise, some patients might not be in need of adalimumab maintenance therapy after successful induction course. The latter hypothesis is supported by the fact that an undetectable infliximab trough level was a predictor of maintenance of remission after discontinuation of antiTNF therapy in the Infliximab Discontinuation in Crohn's Disease Patients in Stable Remission on Combined Therapy with Immunosuppressors (STORI) trial. ${ }^{38}$ Adalimumab trough levels might have more clinical effect in the case of loss of response rather than continuing clinical remission.

Antibody formation to anti-TNF agents is generally associated with reduced efficacy, and switching to another anti-TNF blocker can be beneficial. In rheumatic diseases, immunogenicity to adalimumab is associated with lower trough levels and efficacy, as well as higher discontinuation rates. ${ }^{39}$ In Crohn's disease, limited data are available to estimate the effect of immunogenicity on adalimumab efficacy. The Leuven cohort described 12 patients who developed antibodies to adalimumab. ${ }^{9}$ Eleven of 12 patients demonstrated a trough level lower than $0.094 \mu \mathrm{g} / \mathrm{mL}$ at least once during follow-up, as well as a lower median trough level throughout the entire follow-up period, and 11 patients discontinued adalimumab therapy. A small retrospective study ( $n=30)$ from the Netherlands found adalimumab antibody formation in $17 \%$ of participants, which correlated with nonresponse to adalimumab. ${ }^{40}$ According to available data, immunogenicity correlates with unfavorable outcomes such as lower trough levels and higher discontinuation rates. However, the presence of antibodies to adalimumab by itself might not be sufficient for a switch in therapy.

Infliximab studies demonstrated that factors such as antibody titers and functionality of drug antibodies in addition to trough levels are probably more relevant to the clinical outcome than the development of drug antibodies by itself. This is illustrated by the observation that three of seven subjects (week 24) and two of seven subjects (week 56) who developed antibodies to adalimumab in the CLASSIC II trial were in remission during follow-up. ${ }^{11}$ Further studies regarding the relevance of adalimumab antibodies for clinical outcome and optimization of therapy are needed in the near future. However, if in the case of secondary nonresponse to adalimumab, immunogenicity is combined with low to undetectable trough levels, switching to another anti-TNF agent is frequently required. It should be noted that only indirect evidence from infliximab studies is available to guide this decision. The reverse switch is effective, as shown in patients switching from infliximab to adalimumab because of anti-infliximab antibodies combined with low trough levels. ${ }^{33}$ If adalimumab was the first anti-TNF agent for an individual patient, infliximab is a logical alternative. However, a significant percentage of patients failed infliximab before starting adalimumab. Switching to a third antiTNF agent, certolizumab, can be of benefit. Prior primary nonresponse to anti-TNF therapy and persistent disease activity after 3 months of therapy with the third anti-TNF agent predict decreased efficacy of this switch. ${ }^{41}$ In most countries, only infliximab and adalimumab are approved for the treatment of Crohn's disease, and the lack of approval by regulating authorities often does not allow for a switch to a third anti-TNF agent.

Recent data suggest that infliximab retreatment is feasible in this case. In a series of 29 patients failing adalimumab after prior infliximab use who subsequently restarted infliximab, 93\% experienced sustained clinical benefit after 3 months. ${ }^{42}$ After 18 months, 62\% continued infliximab treatment. Two patients experienced anaphylactic reactions.

If adalimumab trough levels are within a therapeutic window, it is questionable whether dose escalation will 
provide clinical benefit in secondary nonresponders. ${ }^{15,43}$ Anti-TNF therapy in this scenario is not able to control intestinal inflammation, possibly because of a switch in the mechanism of inflammation. ${ }^{44}$ As a consequence, a switch to an alternative anti-TNF agent is less likely to result in clinical benefit. A study from the Mayo Clinic evaluated all Crohn's disease patients who underwent measurement of infliximab drug levels and antibodies to infliximab. ${ }^{33}$ Fifty-one patients demonstrated therapeutic infliximab trough levels, $62 \%$ had no evidence of active inflammation, and for the remaining $38 \%$, various strategies were followed, including infliximab continuation, surgery, switch to another anti-TNF agent, and the addition of immunosuppressive treatment. Unfortunately, no well-designed studies have addressed the issue of active inflammation, despite therapeutic adalimumab drug levels, but most clinicians would not anticipate benefit from adalimumab dose escalation. In these particular situations, switching to an alternative drug class is recommended. In the management of rheumatoid arthritis, switching between drug classes is a well-accepted and effective strategy. For example, switching to rituximab (anti-CD20 monoclonal) or abatacept (anti-CD80/anti-CD86 fusion protein) after anti-TNF failure is used by rheumatologists in clinical practice. ${ }^{45,46}$ Alternative therapies after anti-TNF failure for Crohn's disease include natalizumab, an antibody to $\alpha 4$ integrin, ${ }^{19}$ or agents that await approval by regulating authorities, such as ustekinumab (anti-IL12/IL23) ${ }^{47}$ or vedolizumab (anti- $\alpha 4 \beta 7$ antibody). ${ }^{20}$ Finally, clinical trials can be considered, but up to $70 \%$ of inflammatory bowel disease patients meet exclusion criteria and become ineligible for participation. ${ }^{48}$

Concomitant immunosuppression is thought to increase the efficacy of anti-TNF therapy. Crohn's disease patients in the SONIC trial who were naïve to immunomodulators or anti-TNF therapy achieved higher rates of clinical remission when receiving combination therapy rather than monotherapy. ${ }^{31}$ However, both the CLASSIC II trial and the CHARM trial did not show differences regarding adalimumab levels and remission rates in patients with or without concomitant immunosuppression. ${ }^{12,32}$ Similarly, the Leuven adalimumab cohort did not show differences in remission rates, adalimumab trough levels, or immunogenicity when stratified for concomitant immunosuppression. ${ }^{9}$ Finally, when assessed per semester of maintenance therapy, combination therapy with adalimumab and immunosuppressive therapy did not result in a reduction in semesters with flares compared with adalimumab monotherapy. ${ }^{49}$ So far, data are lacking to support the concept that concomitant immunosuppression will improve therapeutic efficacy of adalimumab therapy.
Some patients will not qualify for or do not respond to the therapeutic options of adalimumab dose escalation or switching within or to another drug class. In these individual cases, surgical intervention should be considered. In specific cases such as patients with small areas of inflammation, a limited resection can be preferred over continuing medical treatment. However, this approach is dependent on individual surgical history, comorbidity, age, and patient preference.

\section{Summary and conclusion}

The majority of Crohn's disease patients will show initial clinical benefit from adalimumab treatment, but failure resulting from nonresponse or adverse events is commonly observed. In the case of adalimumab failure, clinical assessment is required to confirm inflammatory disease activity and rule out noninflammatory causes. Evaluation should include biomarkers (fecal calprotectin and serum CRP), colonoscopy, and/or magnetic resonance enterography/ enteroclysis. Furthermore, adalimumab trough levels and antibodies to adalimumab should be evaluated in these situations. In the case of low or undetectable adalimumab trough levels, dose escalation to $40 \mathrm{mg}$ weekly is recommended, whereas high antibody titers or adverse events frequently result in switching to an alternative anti-TNF agent such as infliximab. Active inflammation despite therapeutic adalimumab trough levels requires alternative strategies such as switching to drugs with a different mode of action or surgical intervention.

One area of future research includes the use of trough levels and antibody formation to affect optimization of adalimumab treatment. Hence, well-designed prospective trials that focus on this particular issue are needed. Furthermore, novel drugs with alternative modes of action are warranted for Crohn's disease patients who fail anti-TNF therapy. Vedolizumab (a therapeutic antibody directed against $\alpha 4 \beta 7$ integrin on lymphocytes) and ustekinumab (an antibody that targets interleukin 12 and interleukin 23) will soon find their way into daily practice and will likely offer significant therapeutic alternatives for the management of these patients. Rheumatoid arthritis serves as a model in which the availability of drugs with distinct working mechanisms allows treating physicians to switch therapies if needed and, more important, to tailor or combine treatment strategies to individual characteristics. For now, optimization of adalimumab therapy before switching to alternative therapies in Crohn's disease patients remains mandatory to fully use the limited therapeutic options currently available. 


\section{Disclosure}

Mark Löwenberg has served as speaker for Abbott/Abbvie, Dr. Falk, Ferring Pharmaceuticals, Merck Sharp \& Dohme and Tramedico. Nanne KH de Boer has served as speaker for Abbott/Abbvie and Merck Sharp \& Dohme; Frank Hoentjen: no disclosures.

\section{References}

1. Frøslie KF, Jahnsen J, Moum BA, Vatn MH; IBSEN Group. Mucosal healing in inflammatory bowel disease: results from a Norwegian population-based cohort. Gastroenterology. 2007;133(2):412-422.

2. van Dullemen HM, van Deventer SJ, Hommes DW, et al. Treatment of Crohn's disease with anti-tumor necrosis factor chimeric monoclonal antibody (cA2). Gastroenterology. 1995;109(1):129-135.

3. Burness CB, Keating GM. Adalimumab: a review of its use in the treatment of patients with ulcerative colitis. Bio Drugs. 2013;27(3):247-262.

4. Hanauer SB, Sandborn WJ, Rutgeerts P, et al. Human anti-tumor necrosis factor monoclonal antibody (adalimumab) in Crohn's disease: the CLASSIC-I trial. Gastroenterology. 2006;130(2):323-333.

5. Pouw MF, Krieckaert CL, Nurmohamed MT, et al. Key findings towards optimising adalimumab treatment: the concentration-effect curve. Ann Rheum Dis. Epub December 10, 2013.

6. Wong DR, Pierik M, Seinen ML, et al. The pharmacokinetic effect of adalimumab on thiopurine metabolism in Crohn's disease patients. J Crohns Colitis. 2014;8(2):120-128.

7. Baert F, Noman M, Vermeire S, et al. Influence of immunogenicity on the long-term efficacy of infliximab in Crohn's disease. $N$ Engl J Med. 2003;348(7):601-608.

8. Sandborn WJ, Rutgeerts P, Enns R, et al. Adalimumab induction therapy for Crohn disease previously treated with infliximab: a randomized trial. Ann Intern Med. 2007;146(12):829-838.

9. Karmiris K, Paintaud G, Noman M, et al. Influence of trough serum levels and immunogenicity on long-term outcome of adalimumab therapy in Crohn's disease. Gastroenterology. 2009;137(5):1628-1640.

10. Roblin X, Marotte H, Rinaudo M, et al. Association between pharmacokinetics of adalimumab and mucosal healing in patients with inflammatory bowel diseases. Clin Gastroenterol Hepatol. 2014;12(1): 80-84.

11. Sandborn WJ, Hanauer SB, Rutgeerts P, et al. Adalimumab for maintenance treatment of Crohn's disease: results of the CLASSIC II trial. Gut. 2007;56(9):1232-1239.

12. Colombel JF, Sandborn WJ, Rutgeerts P, et al. Adalimumab for maintenance of clinical response and remission in patients with Crohn's disease: the CHARM trial. Gastroenterology. 2007;132(1):52-65.

13. Colombel JF, Schwartz DA, Sandborn WJ, et al. Adalimumab for the treatment of fistulas in patients with Crohn's disease. Gut. 2009;58(7): 940-948.

14. Dewint P, Hansen BE, Verhey E, et al. Adalimumab combined with ciprofloxacin is superior to adalimumab monotherapy in perianal fistula closure in Crohn's disease: a randomised, double-blind, placebo controlled trial (ADAFI). Gut. 2014;63(2):292-299.

15. Yanai H, Hanauer SB. Assessing response and loss of response to biological therapies in IBD. Am J Gastroenterol. 2011;106(4):685-698.

16. D'Haens GR, Panaccione R, Higgins PD, et al. The London Position Statement of the World Congress of Gastroenterology on Biological Therapy for IBD with the European Crohn's and Colitis Organization: when to start, when to stop, which drug to choose, and how to predict response? Am J Gastroenterol. 2011;106(2):199-212.

17. Allez M, Karmiris K, Louis E, et al. Report of the ECCO pathogenesis workshop on anti-TNF therapy failures in inflammatory bowel diseases: definitions, frequency and pharmacological aspects. J Crohns Colitis. 2010;4(4):355-366.

18. Siegel CA, Melmed GY. Predicting response to Anti-TNF Agents for the treatment of crohn's disease. Therap Adv Gastroenterol. 2009;2(4): $245-251$.
19. Sandborn WJ, Colombel JF, Enns R, et al; International Efficacy of Natalizumab as Active Crohn's Therapy (ENACT-1) Trial Group; Evaluation of Natalizumab as Continuous Therapy (ENACT-2) Trial Group. Natalizumab induction and maintenance therapy for Crohn's disease. N Engl J Med. 2005;353(18):1912-1925.

20. Sandborn WJ, Feagan BG, Rutgeerts P, et al; GEMINI 2 Study Group. Vedolizumab as induction and maintenance therapy for Crohn's disease. N Engl J Med. 2013;369(8):711-721.

21. Ho GT, Smith L, Aitken S, et al. The use of adalimumab in the management of refractory Crohn's disease. Aliment Pharmacol Ther. 2008;27(4):308-315.

22. Allez M, Vermeire S, Mozziconacci N, et al. The efficacy and safety of a third anti-TNF monoclonal antibody in Crohn's disease after failure of two other anti-TNF antibodies. Aliment Pharmacol Ther. 2010;31(1): 92-101.

23. Chaparro M, Andreu M, Barreiro-de Acosta M, et al. Effectiveness of infliximab after adalimumab failure in Crohn's disease. World $J$ Gastroenterol. 2012;18(37):5219-5224.

24. Hanauer SB, Feagan BG, Lichtenstein GR, et al; ACCENT I Study Group. Maintenance infliximab for Crohn's disease: the ACCENT I randomised trial. Lancet. 4 2002;359(9317):1541-1549.

25. Schreiber S, Khaliq-Kareemi M, Lawrance IC, et al; PRECISE 2 Study Investigators. Maintenance therapy with certolizumab pegol for Crohn's disease. N Engl J Med. 2007;357(3):239-250.

26. Jones J, Loftus EV Jr, Panaccione R, et al. Relationships between disease activity and serum and fecal biomarkers in patients with Crohn's disease. Clin Gastroenterol Hepatol. 2008;6(11):1218-1224.

27. Henriksen M, Jahnsen J, Lygren I, et al; IBSEN Study Group. C-reactive protein: a predictive factor and marker of inflammation in inflammatory bowel disease. Results from a prospective population-based study. Gut. 2008;57(11):1518-1523.

28. Kiss LS, Szamosi T, Molnar T, et al; Hungarian IBD Study Group. Early clinical remission and normalisation of CRP are the strongest predictors of efficacy, mucosal healing and dose escalation during the first year of adalimumab therapy in Crohn's disease. Aliment Pharmacol Ther. 2011;34(8):911-922.

29. Mao R, Xiao YL, Gao X, et al. Fecal calprotectin in predicting relapse of inflammatory bowel diseases: a meta-analysis of prospective studies. Inflamm Bowel Dis. 2012;18(10):1894-1899.

30. Molander P, af Björkesten CG, Mustonen H, et al. Fecal calprotectin concentration predicts outcome in inflammatory bowel disease after induction therapy with TNF $\alpha$ blocking agents. Inflamm Bowel Dis. 2012;18(11):2011-2017.

31. Colombel JF, Sandborn WJ, Reinisch W, et al; SONIC Study Group. Infliximab, azathioprine, or combination therapy for Crohn's disease. N Engl J Med. 2010;362(15):1383-1395.

32. Chiu YL, Rubin DT, Vermeire S, et al. Serum adalimumab concentration and clinical remission in patients with Crohn's disease. Inflamm Bowel Dis. 2013;19(6):1112-1122.

33. Afif W, Loftus EV Jr, Faubion WA, et al. Clinical utility of measuring infliximab and human anti-chimeric antibody concentrations in patients with inflammatory bowel disease. Am J Gastroenterol. 2010;105(5): $1133-1139$.

34. Lopez A, Billioud V, Peyrin-Biroulet C, Peyrin-Biroulet L. Adherence to anti-TNF therapy in inflammatory bowel diseases: a systematic review. Inflamm Bowel Dis. 2013;19(7):1528-1533.

35. Sandborn WJ, Colombel JF, Schreiber S, et al. Dosage adjustment during long-term adalimumab treatment for Crohn's disease: clinical efficacy and pharmacoeconomics. Inflamm Bowel Dis. 2011;17(1): $141-151$.

36. Billioud V, Sandborn WJ, Peyrin-Biroulet L. Loss of response and need for adalimumab dose intensification in Crohn's disease: a systematic review. Am J Gastroenterol. 2011;106(4):674-684.

37. Baert F, Glorieus E, Reenaers C, et al; BIRD (Belgian IBD Research and Development). Adalimumab dose escalation and dose de-escalation success rate and predictors in a large national cohort of Crohn's patients. J Crohns Colitis. 2013;7(2):154-160. 
38. Louis E, Mary JY, Vernier-Massouille G, et al; Groupe D'etudes Thérapeutiques Des Affections Inflammatoires Digestives. Maintenance of remission among patients with Crohn's disease on antimetabolite therapy after infliximab therapy is stopped. Gastroenterology. 2012;142(1):63-70.

39. Mok CC, van der Kleij D, Wolbink GJ. Drug levels, anti-drug antibodies, and clinical efficacy of the anti-TNF $\alpha$ biologics in rheumatic diseases. Clin Rheumatol. 2013;32(10):1429-1435.

40. West RL, Zelinkova Z, Wolbink GJ, et al. Immunogenicity negatively influences the outcome of adalimumab treatment in Crohn's disease. Aliment Pharmacol Ther. 2008;28(9):1122-1126.

41. de Silva PS, Nguyen DD, Sauk J, Korzenik J, YajnikV, AnanthakrishnanAN. Long-term outcome of a third anti-TNF monoclonal antibody after the failure of two prior anti-TNFs in inflammatory bowel disease. Aliment Pharmacol Ther. 2012;36(5):459-466.

42. Brandse JF, Peters CP, Gecse KB, et al. Effects of infliximab retreatment after consecutive discontinuation of infliximab and adalimumab in refractory Crohn's disease. Inflamm Bowel Dis. 2014;20(2): 251-258.

43. Ben-Horin S, Kopylov U, Chowers Y. Optimizing anti-TNF treatments in inflammatory bowel disease. Autoimmun Rev. 2014;13(1):24-30.

44. Chowers Y, Sturm A, Sans M, et al. Report of the ECCO workshop on anti-TNF therapy failures in inflammatory bowel diseases: biological roles and effects of TNF and TNF antagonists. J Crohns Colitis. 2010;4(4):367-376.
45. Finckh A, Ciurea A, Brulhart L, et al; Physicians of the Swiss Clinical Quality Management Program for Rheumatoid Arthritis. B cell depletion may be more effective than switching to an alternative anti-tumor necrosis factor agent in rheumatoid arthritis patients with inadequate response to anti-tumor necrosis factor agents. Arthritis Rheum. 2007;56(5):1417-1423.

46. Genovese MC, Becker JC, Schiff M, et al. Abatacept for rheumatoid arthritis refractory to tumor necrosis factor alpha inhibition. $N$ Engl J Med. 2005;353(11):1114-1123.

47. Sandborn WJ, Gasink C, Gao LL, et al; CERTIFI Study Group. Ustekinumab induction and maintenance therapy in refractory Crohn's disease. N Engl J Med. 2012;367(16):1519-1528.

48. Ha C, Ullman TA, Siegel CA, Kornbluth A. Patients enrolled in randomized controlled trials do not represent the inflammatory bowel disease patient population. Clin Gastroenterol Hepatol. 2012;10(9): 1002-1007.

49. Reenaers C, Louis E, Belaiche J, Seidel L, Keshav S, Travis S. Does co-treatment with immunosuppressors improve outcome in patients with Crohn's disease treated with adalimumab? Aliment Pharmacol Ther. 2012;36(11-12):1040-1048.
Clinical and Experimental Gastroenterology

\section{Publish your work in this journal}

Clinical and Experimental Gastroenterology is an international, peerreviewed, open access journal, publishing all aspects of gastroenterology in the clinic and laboratory, including: Pathology, pathophysiology of gastrointestinal disease; Investigation and treatment of gastointestinal disease; Pharmacology of drugs used in the alimentary tract;

\section{Dovepress}

Immunology/genetics/genomics related to gastrointestinal disease. This journal is indexed on CAS. The manuscript management system is completely online and includes a very quick and fair peer-review system. Visit http://www.dovepress.com/testimonials.php to read real quotes from published authors. 